\title{
A 3D-printed, functionally graded soft robot powered by combustion
}

\section{Citation}

Bartlett, N. W., M. T. Tolley, J. T. B. Overvelde, J. C. Weaver, B. Mosadegh, K. Bertoldi, G. M. Whitesides, and R. J. Wood. 2015. "A 3D-Printed, Functionally Graded Soft Robot Powered by Combustion." Science 349 (6244) (July 9): 161-165. doi:10.1126/science.aab0129.

\section{Published Version}

doi:10.1126/science.aab0129

\section{Permanent link}

http://nrs.harvard.edu/urn-3:HUL.InstRepos:25042249

\section{Terms of Use}

This article was downloaded from Harvard University's DASH repository, and is made available under the terms and conditions applicable to Open Access Policy Articles, as set forth at http:// nrs.harvard.edu/urn-3:HUL.InstRepos:dash.current.terms-of-use\#OAP

\section{Share Your Story}

The Harvard community has made this article openly available.

Please share how this access benefits you. Submit a story.

\section{Accessibility}




\title{
Title: A 3D Printed, Functionally Graded \\ Soft Robot Powered by Combustion
}

Authors: Nicholas W. Bartlett ${ }^{1,2 *} \dagger$, Michael T. Tolley ${ }^{3} \dagger$, Johannes T.B. Overvelde ${ }^{1}$, James C. Weaver $^{2}$, Bobak Mosadegh ${ }^{4}$, Katia Bertoldi $^{1}$, George M. Whitesides ${ }^{2,5}$, Robert J. Wood ${ }^{1,2}$

\author{
Affiliations: \\ ${ }^{1}$ School of Engineering and Applied Sciences, Harvard University, Cambridge, Massachusetts \\ 02138 . \\ ${ }^{2}$ Wyss Institute for Biologically Inspired Engineering, Harvard University, Cambridge, \\ Massachusetts 02138. \\ ${ }^{3}$ Department of Mechanical and Aerospace Engineering, University of California, San Diego, La \\ Jolla, California 92093. \\ ${ }^{4}$ Dalio Institute of Cardiovascular Imaging, Department of Radiology, New York-Presbyterian \\ Hospital and Weill Cornell Medical College, New York, New York 10021. \\ ${ }^{5}$ Department of Chemistry and Chemical Biology, Harvard University, Cambridge, \\ Massachusetts 02138. \\ *Corresponding author. E-mail: nbartlett@seas.harvard.edu \\ $\dagger$ These authors contributed equally to this work.
}

Abstract: Roboticists have begun to design biologically inspired robots with soft or partially soft bodies, which have the potential to be more robust and adaptable, and safer for human interaction, as compared to traditional rigid robots. However, key challenges in the design and manufacture of soft robots include the complex fabrication processes and the interfacing of soft and rigid components. We employed multi-material 3D printing to manufacture a combustionpowered robot whose body transitions from a rigid core to a soft exterior. This stiffness gradient, spanning three orders of magnitude in modulus, enables reliable interfacing between rigid driving components (controller, battery, etc.) and the primarily soft body, and also enhances performance. Powered by the combustion of butane and oxygen, this robot is able to perform untethered jumping.

One Sentence Summary: Interfacing of soft and rigid components through a gradient of material properties increases the robustness of an untethered, jumping soft robot powered by combustion.

\section{Main Text:}

Robots are typically composed of rigid components to promote high precision and controllability. Frequently constructed from hard metals such as aluminum and steel, these robots require large machining equipment and an intricate assembly process. In contrast, recent work has explored the possibility of creating soft-bodied robots (1-5) inspired by invertebrates such as cephalopods (6-8) and insect larvae (9), as well as vertebrates including snakes (10) and fish (11). The use of compliant materials facilitates the development of biologically inspired robotic 
systems (12) that are more adaptable $(13)$, safer $(14,15)$, and more resilient (16) than their fully rigid counterparts.

The design and fabrication of soft robotic systems, however, presents significant engineering challenges $(17,18)$. The bodies of soft robots are typically fabricated in custom-designed molds and require multiple assembly steps (19) or lost-wax techniques (20) to embed actuation. The molds used to create these soft robots are complex and time consuming to make, especially for prototype designs that are fabricated in small numbers and are constantly evolving. Additionally, some applications (e.g. ones requiring untethered robots) necessitate rigid components to power and control the soft body $(10,11,16)$, or to perform specific tasks. The interfaces between these rigid components and the soft body of the robot are points of recurring failure.

In nature, many animals employ stiffness gradients to join rigid materials and soft structures while minimizing stress concentrations that could lead to failures at rigid/soft interfaces $(21,22)$. One of the reasons biological systems often outperform engineered systems is that in nature, which employs self-organization for fabrication, added structural complexity comes at a minimal cost. Emerging digital fabrication technologies (e.g. 3D printing) are beginning to allow designers to move towards this level of structural complexity, albeit at a larger scale and with fewer materials. These technologies can be used to manufacture geometrically intricate designs as efficiently as simple designs with an equivalent amount of material.

We used a multimaterial 3D printer (Connex500, Stratasys Ltd.) to directly print the functional body of a robot that employs soft material components for actuation, obviating the need for complex molding techniques or assembly (23). The robot body is composed primarily of two nested hemispheroids. The flexible bottom hemispheroid features a small depression that provides an initial volume into which oxygen and butane are injected. Ignition of the gases causes a volumetric expansion $(24,25)$, launching the robot into the air (Fig. 1, A and B). The top hemispheroid has a modulus of elasticity that ranges over three orders of magnitude (from approximately $1 \mathrm{MPa}$ to $1 \mathrm{GPa}$ ) through a step-wise gradient of nine different layers, creating a structure that transitions from highly flexible (rubber-like) to fully rigid (thermoplastic-like). In addition to providing a mechanical interface for the rigid control components, the rigid portion of the top hemispheroid also prevents undesired expansion locally and focuses the energy of combustion into the ground, enhancing the jumping efficiency. Pneumatic legs, which use a nested hemi-ellipsoid design similar to that of the main body, surround the central explosive actuator and are used to tilt the body prior to a jump, controlling the direction of locomotion. This separation of power and control actuators simplifies actuation and gives greater control over direction.

In order to simplify prototyping, we chose a modular design with a rigid core module containing the control components (which are expensive and change infrequently during design iteration of the body), connected through a predefined interface to the body of the robot (Fig. 1C). This modularity enables efficient iteration of the robot body design, as well as rapid replacement in the case of destructive testing. The core module contains a custom circuit board, high voltage power source, battery, miniature air compressor, butane fuel cell, bank of six solenoid valves, oxygen cartridge, pressure regulator, and an internal network of channels to facilitate interfacing between the components as necessary (Fig. S1, A and B). The core module is mechanically attached to the rigid portion of the body with a layer of high-strength mushroom-head fasteners. Otherwise, it interfaces with the body only through four tubes (three pneumatic tubes for the legs 
and one tube for fuel delivery to the combustion chamber) and two wires (which produce the spark in the combustion chamber).

Characterization of nine 3D printed materials with a set of mechanical tests informed the design of the $3 \mathrm{D}$ printed rigid/soft robot. We performed qualitative twisting experiments to gain an intuitive understanding of the response of the various materials (Fig. 2A). Mechanical testing on a universal testing machine (Instron 5544, Instron) yielded quantitative values of material properties (supplementary online text). This information was used to simulate the operation of the robot using finite element analysis (FEA) software, which allowed us to compare the relative efficiency of jumping robots with different material distributions. Further simulations allowed us to examine the differences in stress concentrations as a function of material distribution (Fig. S2). While a perfectly smooth gradient from rigid to flexible would have been ideal, the capability of the fabrication technique was limited to a step-wise gradient of at most nine materials. The actuation strategy necessitated a flexible bottom hemispheroid while the off-theshelf control components required a rigid housing; however the stiffness distribution of the top hemispheroid was unconstrained. Thus, to determine how the material properties of the top hemispheroid would affect jumping, we simulated three cases: 1) a flexible top with a small rigid portion to mount control hardware, 2) a top featuring a stiffness gradient from fully flexible to fully rigid, and 3) a fully rigid top (Fig. 2B and Movie S1). Simulations showed that the flexible top was inefficient at directing the energy of combustion into the ground and propelling the robot, suggesting weak jump performance. As expected, the simulated rigid top robot produced the highest ground reaction force, while the gradient top robot exhibited a performance between the two extremes.

We carried out additional simulations to investigate the behavior of the three designs during the impact of landing (Fig. 2C and Movie S1). The results indicate that the rigid top robot experiences a given reaction force (e.g. $50 \mathrm{~N}$, or $11.2 \mathrm{lbf}$ ) at a much smaller deformation than either the gradient or flexible top robots. Immediately upon impact, the rigid top robot experiences an abrupt increase in force, whereas the gradient top robot experiences a more moderate increase. The flexible top robot sees almost no increase, until the small rigid portion impacts the ground, initiating a rapid increase akin to that of the rigid top robot. Integrating the force-displacement curves (up to $50 \mathrm{~N}$ ), we find that the rigid and flexible top robots only absorb $13 \%$ and $73 \%$ (respectively) of the impact energy that the gradient top robot absorbs. The increased energy absorbed by the gradient top robot during impact suggests that it will be most successful at distributing the impulse over a longer duration, therefore reducing peak stresses and providing the least violent landing.

By 3D printing different test cases, we experimentally verified these simulation results. A jumping robot with a completely rigid top was able to jump 1.12 meters untethered using $40 \mathrm{~mL}$ of butane and $120 \mathrm{~mL}$ of oxygen. Identical testing conditions on a gradient top robot produced a jump of 0.25 meters. A flexible top robot was deemed impractical to print due to the predictions from FEA. As predicted by the simulations, the gradient top robot was less efficient at jumping. However, the gradient top robot was better able to withstand the impact of landing (Fig. 3A and Movie S2). In one test, the body of the rigid top robot shattered upon landing, surviving a total of just five jumps; the gradient top robot survived more than twice that number of jumps, and remained operational. Other nearly identical gradient top robots survived over 100 jumps (note: in $81 \%$ of these tests, we removed the core module from the body and delivered the combustion products and ignition sparks through a tether to simplify testing, reducing the system mass to 
about $50 \%$ that of the untethered system). To provide a direct comparison in landing behavior, the gradient top robot was additionally dropped from the maximum height achieved by the rigid top robot, and successfully survived 35 falls (supplementary online text). The stiffness gradient provides the necessary rigidity to transfer the impulse of combustion to generate effective jumping, while the compliance of the base absorbs and dissipates the energy of the landing impact. By trading the jumping efficiency of the rigid robot for an improved ability to survive landings, the gradient top robot demonstrated a greater overall robustness.

Further testing on the gradient top robot showed high resilience and good performance (Fig. 3B and Movie S2). This robot autonomously jumped up to $0.76 \mathrm{~m}$ ( 6 body heights) high, and demonstrated directional jumping of up to $0.15 \mathrm{~m}$ ( 0.5 body lengths, $20 \%$ of jump height $)$ laterally per jump (Fig. 3C and Movie S2). Unlike previous combustion-powered soft jumpers that were either tethered (24) or achieved only a few untethered jumps due to inconsistent connection of electrical and mechanical components at the interface of the rigid and soft components (25), this design allowed for many successful jumps with a single soft robot (21 untethered jumps, 89 tethered jumps). Another jumper design has also shown the ability to perform multiple jumps, can operate on uneven terrain, and can even recover from landing in any orientation (26), although at the sacrifice of directional control. In our system, the high energy density of the fuels theoretically allows onboard storage of sufficient fuel for 32 consecutive jumps (supplementary online text). The bodies were extremely robust, surviving dozens of jumps before they became unusable. The monolithic design has no sliding parts or traditional joints that can be fouled or obstructed by debris or rough terrain, and the nested design requires minimal deformation for actuation. As with previous jumping soft robots powered by combustion $(24,25$, 26), and untethered systems exposed to direct flames (16), we did not observe significant damage to the soft (or rigid) body materials due to the brief exposure to elevated combustion temperatures and flames.

The fabrication of soft robots using multi-material 3D printing has numerous advantages over traditional molding techniques. This strategy promotes high throughput prototyping by enabling rapid design iteration with no additional cost for increased morphological complexity. By allowing designers greater freedom, 3D printing also facilitates the implementation of good robotic design principles, such as modularity and the separation of power and control actuators. Beyond soft robotics specifically, the ability to print a single structure composed of multiple materials enables investigation into mechanically complex designs, without the drawbacks of complicated assembly or inconsistent manufacturing repeatability. One such design is a modulus gradient that eases the transition from soft to rigid components through stress reduction at the interface of materials mismatched in compliance. Although the materials available to this fabrication strategy are currently limited, and perhaps best suited to the fabrication of prototype devices, future development of materials compatible with 3D printing will only enhance the relevance of this technique.

\section{References and Notes}

1. D. Trivedi, C. D. Rahn, W. M. Kier, I. D. Walker, Applied Bionics and Biomechanics 5, 99 (2008).

2. R. Pfeifer, M. Lungarella, F. Iida, Communications of the ACM 55, 76 (2012).

3. S. Kim, C. Laschi, B. Trimmer, Trends in biotechnology 31, 287 (2013). 
4. C. Majidi, Soft Robotics 1, 5 (2014).

5. C. Laschi, M. Cianchetti, Frontiers in bioengineering and biotechnology 2 (2014).

6. W. McMahan, et al., Robotics and Automation, 2006. ICRA 2006. Proceedings 2006 IEEE International Conference on (IEEE, 2006), pp. 2336-2341.

7. R. F. Shepherd, et al., Proceedings of the National Academy of Sciences 108, 20400 (2011).

8. C. Laschi, et al., Advanced Robotics 26, 709 (2012).

9. H.-T. Lin, G. G. Leisk, B. Trimmer, Bioinspiration \& biomimetics 6, 026007 (2011).

10. C. D. Onal, D. Rus, Bioinspiration \& biomimetic 8, 026003 (2013).

11. A. D. Marchese, C. D. Onal, D. Rus, Soft Robotics 1, 75 (2014).

12. R. Pfeifer, M. Lungarella, F. Iida, Science 318, 1088 (2007).

13. K. Suzumori, S. Iikura, H. Tanaka, Control Systems, IEEE 12, 21 (1992).

14. Y.-L. Park, et al., Bioinspiration \& biomimetics 9, 016007 (2014).

15. P. Polygerinos, Z. Wang, K. C. Galloway, R. J. Wood, C. J. Walsh, Robotics and Autonomous Systems, in press (available at http://www.sciencedirect.com/science/article/pii/S0921889014001729).

16. M. T. Tolley, et al., Soft Robotics 1, 213 (2014).

17. H. Lipson, Soft Robotics 1, 21 (2014).

18. K.-J. Cho, et al., International Journal of Precision Engineering and Manufacturing 10, 171 (2009).

19. F. Ilievski, A. D. Mazzeo, R. F. Shepherd, X. Chen, G. M. Whitesides, Angewandte Chemie 123, 1930 (2011).

20. E. Steltz, A. Mozeika, N. Rodenberg, E. Brown, H. M. Jaeger, Intelligent Robots and Systems, 2009. IROS 2009. IEEE/RSJ International Conference on (IEEE, 2009), pp. 56725677.

21. T. J. Roberts, E. Azizi, The Journal of Experimental Biology 214, 353 (2011).

22. A. Miserez, T. Schneberk, C. Sun, F. W. Wok, J. H. Waite, Science 319, 1867 (2008).

23. Materials and methods are available as supplementary materials on Science Online.

24. R. F. Shepherd, et al., Angewandte Chemie 125, 2964 (2013).

25. M. T. Tolley, et al., Intelligent Robots and Systems (IROS 2014), 2014 IEEE/RSJ International Conference on (IEEE, 2014), pp. 561-566.

26. M. Loepfe, C. M. Schumacher, U. B. Lustenberger, W. J. Stark, Soft Robotics 2, 33 (2015).

27. R. W. Ogden, Non-linear elastic deformations (Courier Corporation, 1997).

28. E. D. Reedy, T. R. Guess, International Journal of Solids and Structures 30, 2929 (1993). 
Acknowledgments: This material is based on work supported by the National Science Foundation under award number DMR-0820484, the Wyss Institute for Biologically Inspired Research, and the Army Research Office, National Defense Science and Engineering Graduate (NDSEG) Fellowship. Any opinions, findings, conclusions, or recommendations expressed in this material are those of the authors and do not necessarily reflect those of the funding organizations.

\author{
Supplementary Materials \\ www.sciencemag.org \\ Materials and Methods \\ Supplementary Text \\ Figures S1 and S2 \\ Table S1 \\ Movies S1 and S2 \\ References $(27,28)$
}

Fig. 1. Robot design and principle of operation. (A) To initiate a jump, the robot inflates a subset of its legs to tilt the body in the intended jump direction. Upon combustion, the bottom hemispheroid balloons out, pushing against the ground and propelling the robot into the air. (B) The ignition sequence consists of fuel delivery, mixing, and sparking. Butane and oxygen are alternately delivered to the combustion chamber (to promote mixing). After a short delay to promote additional mixing of the fuels, the gaseous mixture is ignited, resulting in combustion. Leg inflation occurs concurrently with fuel delivery, and leg deflation begins shortly after landing. (C) Computer aided design (CAD) model of the entire robot consisting of the main explosive actuator surrounded by three pneumatic legs. A rigid core module that contains power and control components sits atop the main body, protected by a semi-soft shield.

Fig. 2. Material tests and simulation results. (A) Qualitative twisting analysis comparing 3D printed beams that are fully flexible, half rigid and half flexible, or transition gradually from rigid to flexible. These tests were performed to gain an intuition as to how these materials respond, as well as to validate the numerical values of the material properties used in simulation. (Left) Material distribution of the beams. (Middle) Beams under torsion. (Right) Simulation of beams under torsion. (B) Jumping simulation. (Left) Ground reaction force as internal gases expand. (Middle) Pressure evolution inside the robot body as internal gases expand. (Right) Deformation state of rigid top, gradient top, and flexible top robot bodies at the initial state and the point of maximum simulated gas expansion. Line thicknesses indicate material stiffness. (C) Impact simulation. In the simulation, the robot impacts the ground at 45 degrees. This angle was chosen as a particularly extreme loading condition, and because it correlated with observations from jumping experiments. (Left) Reaction forces experienced by the three robots upon impacting a solid plane under simulated conditions representative of actual testing conditions. (Right) Finite element analysis (FEA) results of rigid top, gradient top, and flexible top robots, compared at $50 \mathrm{~N}$. 
Fig. 3. Experimental testing results. (A) Frames shortly after the moment of ground contact from Movie S2 (Impact Comparison). Identical testing conditions were used to analyze the difference in landing between a robot with a rigid top and one with a gradient top. Because the rigid top robot jumped higher under combustion powered testing, the gradient top robot was dropped from the maximum height achieved by the rigid top robot for a direct comparison. (Left) The rigid top robot fractures upon impact. (Right) The gradient top robot is able to absorb the impact and survive the fall. (B) Frames from Movie S2 (Jump onto Table) at various times. The robot performs a targeted jump off of an angled surface onto a table. (Left) As the robot prepares for the jump, oxygen and butane are delivered into the combustion chamber. (Middle) Upon ignition of the fuel, the robot is propelled into the air. (Right) After jumping across a gap, the robot lands on a table. (C) Frames from Movie S2 (Directional Jump) at various times during a directional jump. The robot pitches backwards during the jump, providing a soft landing on the inflated legs. Upon impact with the ground, the robot pitches forward and returns to its pre-jump stance. 